\title{
Escolaridad y trabajo en jóvenes rurales. Un estudio etnográfico en San Juan Coyula, Oaxaca
}

\author{
Schooling and work in rural youth. An ethnographic \\ research in San Juan Coyula, Oaxaca
}

Iván Auli

Departamento de Investigaciones Educativas del Centro de Investigación y de Estudios Avanzados, México jairo.auli@cinvestav.mx

ORCID 0000-0002-4802-0065

\begin{abstract}
RESUMEN
El presente artículo condensa resultados de una investigación que analizó los significados de la escolaridad y el trabajo en un grupo de jóvenes rurales mexicanos. El estudio se basó en un enfoque etnográfico; se realizaron entrevistas en profundidad, también se contemplaron observaciones y notas de campo. Los resultados muestran que para estos jóvenes la "convivencia" es el motivo principal para asistir a la escuela. En varios casos es acompañado por "falta de interés en los estudios", por lo que tienden a faltar regularmente a clases; esto se vuelve motivo para que algunos abandonen la escuela. Complementariamente, los jóvenes consideran el trabajo una actividad fundamental, porque les brinda la posibilidad de ayudar a la reproducción familiar, al mismo tiempo que les permite "tener dinero". Para los jóvenes rurales el trabajo es más importante que la escolaridad. Sólo un pequeño grupo manifiesta "interés en los estudios", ya que la escolaridad les sirve para salir adelante y encontrar mejores trabajos; en específico, la intención de las mujeres es superar la condición de género que ocupan en la comunidad. Los hallazgos aportan reflexiones para pensar la formación escolar en contextos rurales.
\end{abstract}

Palabras clave: escolaridad y trabajo, juventud rural, estudiantes, jóvenes, experiencia escolar, México.

\begin{abstract}
This article summarizes results of a research that analyzed the meanings of education and labor in a group of rural young people in Mexico. This research had an ethnographic approach; conducting in-depth interviews and taking into account observations and field notes. The results show that for these young, "seeing friends" is the most important reason for attending school. In several cases, it has to do with "the lack of interest in studying" that causes absenteeism; hence, the result is dropping out of school. Besides, for young people, work is considered fundamental because it allows them to help their families. At the same time, work enables them to "have money". For rural young people, work is more important than schooling. No more than a small group shows "interest in studying" because schooling will help them to get ahead and find better jobs; precisely, rural women aspire to overcome the gender condition they have in their community. The findings provide reflections to think about schooling in rural contexts.
\end{abstract}

Keywords: schooling and work, rural youth, students, young people, school experience, Mexico. 
En memoria de mi mentor, el Dr. Eduardo Weiss, sus consejos fueron fundamentales para ampliar mi horizonte de comprensión.

\section{INTRODUCCIÓN}

En este texto se presentan resultados de una investigación desarrollada entre septiembre de 2016 y agosto de 2018. Su objetivo fue analizar, desde un enfoque etnográfico, la manera en que viven la escolaridad y el trabajo los jóvenes rurales de San Juan Coyula, comunidad ubicada en el estado de Oaxaca, México. ${ }^{1}$

La problemática retoma dos aspectos complementarios. Primero, desde 2012 en México se legisló la obligatoriedad de la Educación Media Superior (EMS), lo que significa un interés por parte del gobierno de aumentar la escolarización de la población, para lo cual se implementaron medidas como aumentar la cobertura, ampliar instalaciones, contratar docentes y establecer nuevas modalidades (como los Telebachilleratos Comunitarios y Preparatoria en Línea). En este contexto, se han realizado investigaciones que abordan temas como el abandono escolar en la EMS (Silva y Weiss, 2018) y la configuración de la acreditación de asignaturas en la cotidianidad de la asistencia al bachillerato (Saccone y Weiss, 2017; Saccone, 2020).

La obligatoriedad de la EMS implica asistir a los sectores rurales. Por ello, la investigación que se presenta aquí aporta un insumo para comprender la manera en que algunos jóvenes rurales se encuentran viviendo este proceso.

Como segundo aspecto, de acuerdo con los indicadores de 2013 a 2016 que presentó el Instituto Nacional para le Evaluación de la Educación (INEE 2013, 2016), se observa que la tasa de absorción de la EMS en Oaxaca es de las más bajas del Sistema Educativo Nacional (SEN). Por lo anterior, se acudió a San Juan Coyula, una pequeña población rural de Oaxaca en la que la mayoría de los jóvenes, al terminar la telesecundaria, deja de lado su escolaridad por empezar a trabajar. En este contexto, se hace pertinente preguntar ¿qué significados y sentidos han construido sobre la escolaridad? Y más específicamente, ¿qué significados y sentidos asignan al trabajo? Cabe señalar que son contados los jóvenes (sobre todo las mujeres)

\footnotetext{
${ }^{1}$ El artículo es resultado de la tesis de maestría del autor titulada Escolaridad y trabajo de jóvenes rurales. Un estudio etnográfico en San Juan Coyula, Oaxaca, en el Departamento de Investigaciones Educativas del Centro de Investigación y de Estudios Avanzados (DIECinvestav), coordinada por el Dr. Eduardo Weiss.
} 
que se muestran interesados en continuar sus estudios en el siguiente nivel educativo; ¿qué lleva a este pequeño grupo de estudiantes a interesarse por la escolaridad? Los resultados que se obtuvieron sirven para la construcción de propuestas políticas y pedagógicas que atiendan la formación de la población rural.

La investigación retomó la perspectiva de los jóvenes. Sin embargo, para obtener un análisis más amplio, se consideró el criterio de los padres de familia.

A continuación, se muestran los aspectos teórico-metodológicos de la investigación. Después se exponen los resultados. Por último, en las conclusiones se plantea una síntesis de los hallazgos, así como reflexiones generales a las que se llegó respecto de la problemática estudiada.

\section{ASPECTOS TEÓRICO-METODOLÓGICOS}

\section{Antecedentes de la investigación}

Con la intención de realizar un trabajo etnográfico con sustento teórico, se revisaron investigaciones previas, entre las que destacan los estudios realizados en la línea de investigación Jóvenes y escuela (Weiss, 2012a), apoyada en el concepto de significado -que aduce a aquellas construcciones colectivas compartidas en un contexto- y sentido -que alude a experiencias individuales-. También se enfoca a los jóvenes desde su triple condición de estudiantes, jóvenes e hijos de familia (Weiss, 2006a y Weiss, 2012b).

En la misma línea de investigación se encuentra el estudio de Guerra y Guerrero (2004), que describe distintos significados que jóvenes urbanos otorgan a la escolaridad. Los significados son "la escolaridad como medio para continuar estudios superiores", "como movilidad económica y social”, "espacio de vida juvenil”, "lugar formativo", "para enfrentar la condición de género", "medio para adquirir autoestima y valoración social”. El estudio muestra que la mayoría de los jóvenes asisten a la escuela para obtener el certificado, ya que tienen la expectativa de realizar estudios superiores. Asimismo, destacó que uno de los significados importantes es la posibilidad de encontrarse con otros jóvenes. Este hallazgo abrió una serie de estudios que analizaron la vida juvenil manifiesta en la escuela (Áva- 
los, 2007; Hernández, 2008; Grijalva, 2010; Vega, 2013), espacio donde lo escolar se convierte en un lugar de expresión, de construcción de relaciones y de identidades juveniles.

Por otro lado, en esta misma línea de investigación se presenta el estudio de Tapia (2015), que explora los significados de jóvenes residentes en una zona en transición rural-urbana. Tapia (2015) menciona que los jóvenes significan al bachillerato y al trabajo como lugares de mejora social, individual y familiar (ser alguien en la vida, salir adelante y ayudar a mi familia). También como desarrollo personal (aprender del trabajo, ser responsables, ser independientes, valerme por mi mismo, aprender y ser mejores). Por último, encuentra la formación escolar profesional (primero tengo mi carrera y trabajar y ahorrar para ir a la universidad). Cabe señalar dos puntos de la investigación de Tapia (2015), quien observó la creciente inclusión de las mujeres al mundo escolar y la presencia fuerte de la dimensión familiar, que se encuentra ligada a una retribución sobre los esfuerzos familiares.

Weiss (2006b) señala que algunos jóvenes rurales e indígenas tienen la expectativa de seguir estudiando. Esto representa un cambio generacional y plantea que, para algunos jóvenes de sectores rurales e indígenas, hay un interés importante en la obtención del certificado, hecho que parece abrir nuevas aspiraciones de trabajo para jóvenes que provienen de familias dedicadas al trabajo agrícola y a la migración temporal a la ciudad para emplearse en trabajos informales.

Otra investigación es la que elaboró Reyes (2010), quien analizó la experiencia estudiantil de adolescentes rurales. Reyes (2010, p. 186) encontró que los jóvenes "desarrollan la capacidad crítica a partir de la cual despliegan una mirada reflexiva sobre la escuela y las otras instituciones de las que forman parte, pero también sobre sí mismos"; ésta es una experiencia que se configura de distintas formas. Algunas son una experiencia "crítica atada al peso de las instituciones y ante un futuro incierto". Estos jóvenes desarrollan la idea de que la escolaridad es importante; sin embargo, su mirada se encuentra atada al peso de la decisión familiar, que por lo regular niega la oportunidad de adquirir mayor escolaridad. Aparece también la experiencia escolar "como paréntesis en proyectos e intereses"; las expectativas de los jóvenes se encuentran al margen de adquirir mayor escolaridad porque la escuela deja de significarse 
como útil. La secundaria es el último nivel escolar que cursarán, por lo que su asistencia es vista principalmente como un espacio de convivencia y diversión juvenil. Por su parte, la experiencia "emotiva pero reflexiva" da cuenta de las relaciones entre pares (amicales o de noviazgo) que se generan en el espacio educativo que los lleva a madurar y a mostrarse reflexivos ante las instituciones y las perspectivas a futuro. En la experiencia estudiantil "recuperada y liberadora", la secundaria se convierte en una oportunidad para construir un futuro diferente del de los otros jóvenes que han quedado marginados, con la intención de dejar a un lado su contexto de carencia económica y de inequidad social. La experiencia "desencantada" se combina con problemas familiares, que en la mayoría de los casos lleva a los jóvenes a abandonar la escuela. Por último, la experiencia "forastera" es construida por estudiantes que se desplazan de zonas urbanas a comunidades rurales, estos jóvenes "se enfrentan a contrastes y tensiones que se crean como producto del encuentro entre el mundo sociocultural en el que se vivía y el nuevo que se habita" (Reyes, 2014, p. 91).

De forma complementaria se revisaron algunos estudios que analizaron específicamente los significados que los jóvenes otorgan al trabajo. En este contexto, encontramos la investigación de Guerra (2005), quien configura cuatro dimensiones del trabajo y en cada una de ellas distribuye nueve categorías de sentido, de la siguiente manera: "el trabajo como un medio para otros fines" (la sobrevivencia familiar, la movilidad familiar, el consumo individual, la independencia residencial y apoyo a los estudios); "el trabajo como norma o tradición" (estar activo mientras pienso qué hacer); "el trabajo y los vínculos afectivos" (búsqueda de sociabilidad) y "trabajo como medio para la formación y estabilidad" (formación profesional y búsqueda de estabilidad laboral con orientación al desarrollo).

En su investigación, Tapia y Weiss (2013) abordaron los significados del trabajo para estudiantes de una zona rural-urbana. Desde la perspectiva de los autores, éstos se encuentran influidos por su asistencia a la escuela, pero principalmente por su horizonte familiar. Los jóvenes atribuyen los siguientes significados al trabajo; "como ayuda para la familia", la cual se encuentra pensada como una especie de reciprocidad o de ayuda mutua. En algunos casos se observa una articulación entre el trabajo y la escuela, ya que la remu- 
neración laboral ayuda a asumir los gastos económicos que implica asistir a la misma. Los jóvenes también mencionan que "el trabajo es una oportunidad de aprendizaje". Por último, "el trabajo como medio para una mayor autonomía personal" es expresado por los jóvenes para indicar que el trabajo contribuye a la construcción de autonomía ante la dependencia familiar.

La revisión de las investigaciones ha sido importante, ya que permitió distanciarse de prejuicios. Además, ayudó a construir categorías para analizar el referente empírico.

\section{UN ENFOQUE ETNOGRÁFICO}

El desarrollo de esta investigación se basó en el enfoque etnográfico. La etnografía de la que se parte corresponde con aquella que se sustenta en la idea de "documentar lo no documentado"; "lo nodocumentado es lo familiar, lo cotidiano, lo oculto, lo inconsciente" (Rockwell, 2009, p. 21). El propósito fue indagar las percepciones y vivencias de los sujetos, "no documentada por los informes burocráticos, pero tampoco por las investigaciones pedagógicas, sociológicas, psicológicas o antropológicas que se acercan con un marco teórico preconcebido" (Weiss, 2012a, p. 27).

De esta manera fue necesario inscribirse en la vida cotidiana de los informantes, vista como el "conjunto de prácticas, relaciones, significaciones diversas y heterogéneas que construyen sujetos particulares al interior de una realidad concreta" (Achilli, 2005, p. 21). En este contexto, se define a la etnografía como una ciencia narrativa que presenta las vivencias y miradas de los sujetos; en el fondo son los protagonistas quienes muestran sus interpretaciones. ${ }^{2}$

\section{Trabajo de campo}

Como se advirtió anteriormente, la investigación se realizó con los jóvenes de San Juan Coyula, Oaxaca, México. ${ }^{3}$ Esta pequeña comu-

\footnotetext{
${ }^{2} \mathrm{El}$ concepto de etnografía al que se llegó se inscribe en la tradición antipositivista que busca interpretar en lugar de explicar (Geertz, 2001).

${ }^{3}$ El estado de Oaxaca se encuentra conformado por ocho regiones. Asimismo, cada región se encuentra dividida en Distritos, dentro de los cuales encontramos los 570 municipios que
} 
nidad rural se encuentra conformada por 719 habitantes $(352 \mathrm{mu}-$ jeres y 367 hombres) distribuidos en 163 viviendas (INEGI, 2010). La mayoría, de entre 30 y 80 años de edad, manifiesta que sólo tienen los primeros tres grados de educación básica, lo que indica que se trata de una población sin primaria completa. El trabajo en el que se ocupan en mayor proporción tiene que ver con labores agropecuarias de subsistencia; siembran maíz de temporal y crían animales de granja. La carestía del campo ha llevado a que las personas migren a la ciudad de México y a Estados Unidos como sus principales destinos. La migración de las familias, a su vez, hace posible la existencia de flujos culturales, ${ }^{4}$ pues incorporan prácticas de otros lugares, al grado de que algunos muestran características de urbanidad. San Juan Coyula es un lugar cuyos habitantes son tradicionales y simultáneamente modernos.

Coyula cuenta con tres escuelas: un preescolar, una primaria y una telesecundaria. Debido a que la comunidad es pequeña, no cuenta con bachillerato, por lo que los jóvenes que quieren estudiar el bachillerato tienen que migrar a la ciudad o trasladarse al pueblo vecino (Cuyamecalco Villa de Zaragoza).

Las estrategias metodológicas que se utilizaron fueron: entrevistas en profundidad (Taylor y Bogdan, 1987), observaciones y notas de campo. Se realizaron 34 entrevistas a 12 alumnos de $3^{\circ}$ de telesecundaria, cuatro egresados de la telesecundaria que no siguieron estudiando el bachillerato, tres que abandonaron la telesecundaria, tres jóvenes que no ingresaron a la telesecundaria, una estudiante de bachillerato, tres que abandonaron el bachillerato y ocho padres de familia. ${ }^{5}$ Las edades de los jóvenes entrevistados oscilan entre los 13 y los 23 años. Por otro lado, las edades de los padres de familia van de los 40 a los 47 años. Las observaciones se hicieron en el salón de $3^{\circ}$ de telesecundaria con la intención de tomar en cuenta ciertas prácticas de los estudiantes. También se realizaron

conforman al estado. La región de la Cañada se encuentra subdividida por dos distritos: Teotitlán de Flores Magón y Cuicatlán; a este último pertenece el Municipio de San Juan Bautista Cuicatlán que alberga la Agencia Municipal de San Juan Coyula.

${ }^{4}$ La noción de flujo cultural va contra la definición de cultura como modelo de conducta estático y específico de una comunidad (Pérez, 2006).

${ }^{5}$ Por cuestiones de presentación y extensión, en la primera mención de un informante se muestra entre paréntesis su situación escolar, después, sólo se alude a su nombre. 
notas de campo, que tienen cierto parecido a un diario, ya que ahí se escribieron detalles observados, así como interpretaciones previas al trabajo de análisis.

\section{El análisis de la información: espiral hermenéutica y descripción densa}

El primer paso que se consideró para el análisis fue la transcripción de entrevistas para categorizar los temas recurrentes. Después, se construyeron matrices generales junto a una colección de frases significativas para comparar las expresiones (similares/diferentes) de los informantes (Weiss, 2017). Simultáneamente, se leían las observaciones y notas de campo. Al contar con matrices generales, se comenzó un diálogo con los antecedentes de la investigación; esto permitió condensar las matrices de acuerdo con categorías analíticas, añadiendo las que surgieron en el trabajo de campo (Glaser y Strauss, 1967).

La espiral hermenéutica fue fundamental. Según Weiss (2017), este proceso se caracteriza por varias vueltas entre deducción e inducción; construcción y reconstrucción de matrices condensadas, que nos lleva de anticipaciones de sentido a una comprensión más lograda.

Al escribir el informe final se utilizó la descripción densa (Geertz, 2001), ya que se redactaron párrafos con datos empíricos. El resultado es una exposición descriptiva con la intención de "conservar la riqueza de las relaciones particulares de la localidad en que se hizo el estudio" (Rockwell, 2009, p. 21).

\section{RESULTADOS DE LA INVESTIGACIÓN}

\section{Significados de la escolaridad y el trabajo}

El cuadro 1 condensa los significados que los jóvenes expresan acerca de la escolaridad y el trabajo; el cuadro se organiza de acuerdo con las categorías analíticas construidas, las cuales contienen los significados concretos expresados por los 26 casos. Asimismo, se agrupa a los jóvenes según su situación escolar.

En el cuadro se aprecia que casi siempre se combinan diferentes significados; los jóvenes manejan diversos significados que orientan 
su acción, éstos suelen ser armónicos, pero también ambiguos. Es posible comprender que los jóvenes realizan un proceso de jerarquización que orienta su acción (Gessaghi y Sendón, 2008); en algunos casos es evidente, en otros existe dificultad para observarla, por lo que es imprescindible considerar detalles (observaciones y notas de campo). Por último, se advierte que las expresiones y formas de actuar provienen de los diferentes contextos en los que participan (casa o escuela) y de las relaciones amicales y familiares que establecen; en el caso de los jóvenes coyuleros, los significados tienen su fundamento principal en la familia.

El cuadro 1 también muestra la frecuencia con la que mencionaron determinado significado. La "convivencia" es la categoría que aparece en los 26 casos; 14 jóvenes mencionan que en la escuela estoy con mis amigos, nueve porque en casa y pueblo me aburro, seis señalan que en casa es trabajo. Por último, el echar relajo aparece en ocho casos. Por su parte, en la "falta de interés en los estudios" diez jóvenes comparten que las clases y temas son aburridos, cuatro que la escuela no me llama la atención y seis que estudiar es en vano. Una minoría de jóvenes, sobre todo mujeres, muestran "interés en los estudios", en esta categoría siete indicaron que la escolaridad ayuda a salir adelante, diez para encontrar mejores trabajos y siete porque en la escuela aprendemos cosas para la vida.

Por otro lado, los significados del trabajo fueron organizados en dos categorías. Según la frecuencia, primero encontramos la categoría para "tener dinero" en el que 14 de los 26 casos expresan que el trabajo les permite comprar cosas. ${ }^{6}$ Por su parte, cinco indican que el trabajo es el medio para mantenerse. Cinco jóvenes manifiestan que el trabajo es importante, ya que permite pagar mis estudios, este significado sólo es referido por mujeres. Por último, en la categoría "ayudar a mi familia", 13 de los 26 jóvenes entrevistados compartieron que el trabajo es importante para apoyar en la reproducción familiar, porque mis papás no pueden solos. Solamente cuatro significaron que el trabajo les brinda la posibilidad de componer la casa de mis papás.

El cuadro 1 evidencia que estar con amigos y las clases y temas son aburridos son los significados predominantes. Por otro lado, los

\footnotetext{
${ }^{6}$ Se hace referencia a la adquisición de bienes materiales como: celular, ropa, zapatos y otros productos.
} 
significados frecuentes del trabajo son para comprar cosas y mis papás no pueden solos.

$\mathrm{El}$ análisis de todas las categorías y significados excede las posibilidades del presente artículo. Al respecto se han seleccionado los casos representativos que ayudan a sustentar las conclusiones y reflexiones a las que se llegó a lo largo del proceso de la investigación.

\section{SIGNIFICADOS Y SENTIDOS DE LA ESCOLARIDAD}

\section{Convivencia}

\section{Estoy con mis amigos}

Los 26 jóvenes entrevistados mencionaron que asisten a la escuela para estar con sus amigos.

Irma (E23-06-2017, alumna de $3^{\circ}$ de telesecundaria) menciona que su papá no la deja tener amigos en la comunidad, porque "sólo quitan el tiempo o siempre nos llevan por el mal camino, por eso, no nos da permiso que salgamos". Irma indica que su mamá las acompaña a todos lados, incluso si se trata de algún trabajo en equipo. Por lo anterior, Irma menciona que "la escuela me gusta, porque puedo estar con mis amigos sin que mis papás me anden vigilando. Ahí ellos no me critican con quién me ando juntando".

Por otro lado, Luis Miguel (E04-04-2017, egresado de la telesecundaria) menciona que fuera de la escuela difícilmente puede estar con todos sus amigos, ya sea porque a algunos no los dejan salir sus papás o porque tienen que ir al campo. Debido a lo anterior, a Luis Miguel le gustaba ir a la secundaria porque "ahí llegaban todos mis amigos y la pasábamos bien, aunque luego nos regañaban por el escándalo y ya nos aquietábamos".

Generalmente, los jóvenes coyuleros no se reúnen por las tardes o lo hacen esporádicamente (fiesta del pueblo o algún evento familiar). En algunas comunidades rurales e indígenas se observa que las canchas de basquetbol o futbol permiten que los jóvenes se reúnan. Sin embargo, la experiencia en Coyula indica que algunos no asisten a ellas porque tienen que trabajar o sus padres no los dejan (sobre todo a las mujeres). En este contexto, la escuela constituye 
la oportunidad para convivir con otros jóvenes con quienes, incluso, construyen relaciones de amistad (N19-03-2017).

El aburrimiento de la casa y el pueblo ha llevado a algunos jóvenes a que la escuela sea un lugar relevante en el que pueden distraerse.

Yesenia (E25-06-2017, alumna de $3^{\circ}$ de telesecundaria) dice que no le gusta salir de su casa, porque no se le hace divertido, "si hay algo como una fiesta o juegos artificiales, tal vez, pero no, no le encuentro chiste. A veces salgo con mis primas, pero, cuando no, no salgo". Por lo que la escuela es uno de sus lugares favoritos para distraerse, "hacemos trabajos y también estamos todos; a veces riendo otras peleando, pero está uno distraído en algo".

En otros casos, como el de Eliseo (E15-06-2017, alumno de $3^{\circ}$ de telesecundaria) la escuela funge como apaciguadora del aburrimiento, ya que en casa "aunque veo la tele y eso, pero se aburre uno". En la escuela Eliseo platica y juega con sus amigos, lo que le permite distraerse.

\section{En casa y en el pueblo me aburro}

El caso de Fabián (E16-06-2017, alumno de $3^{\circ}$ de telesecundaria) es similar al de Eliseo, pero se complementa con que su casa se encuentra a las afueras del pueblo. Fabián expresa que gracias a la escuela no se siente solo: "ahí en mi casa siempre estoy solo, ahí donde vivo casi no hay mucha gente, porque vivo hasta allá arriba [señala el cerro] y aquí en el centro hay más gente”.

La vida en Coyula es rutinaria, por lo que la escuela se vuelve el lugar para evitar el aburrimiento que genera estar en el pueblo, donde hay pocos espacios para el esparcimiento juvenil.

\section{En casa es trabajo}

Los jóvenes coyuleros trabajan cotidianamente con la intención de ayudar en la reproducción familiar, por lo que la escuela es una oportunidad para escapar de esas responsabilidades familiares. 


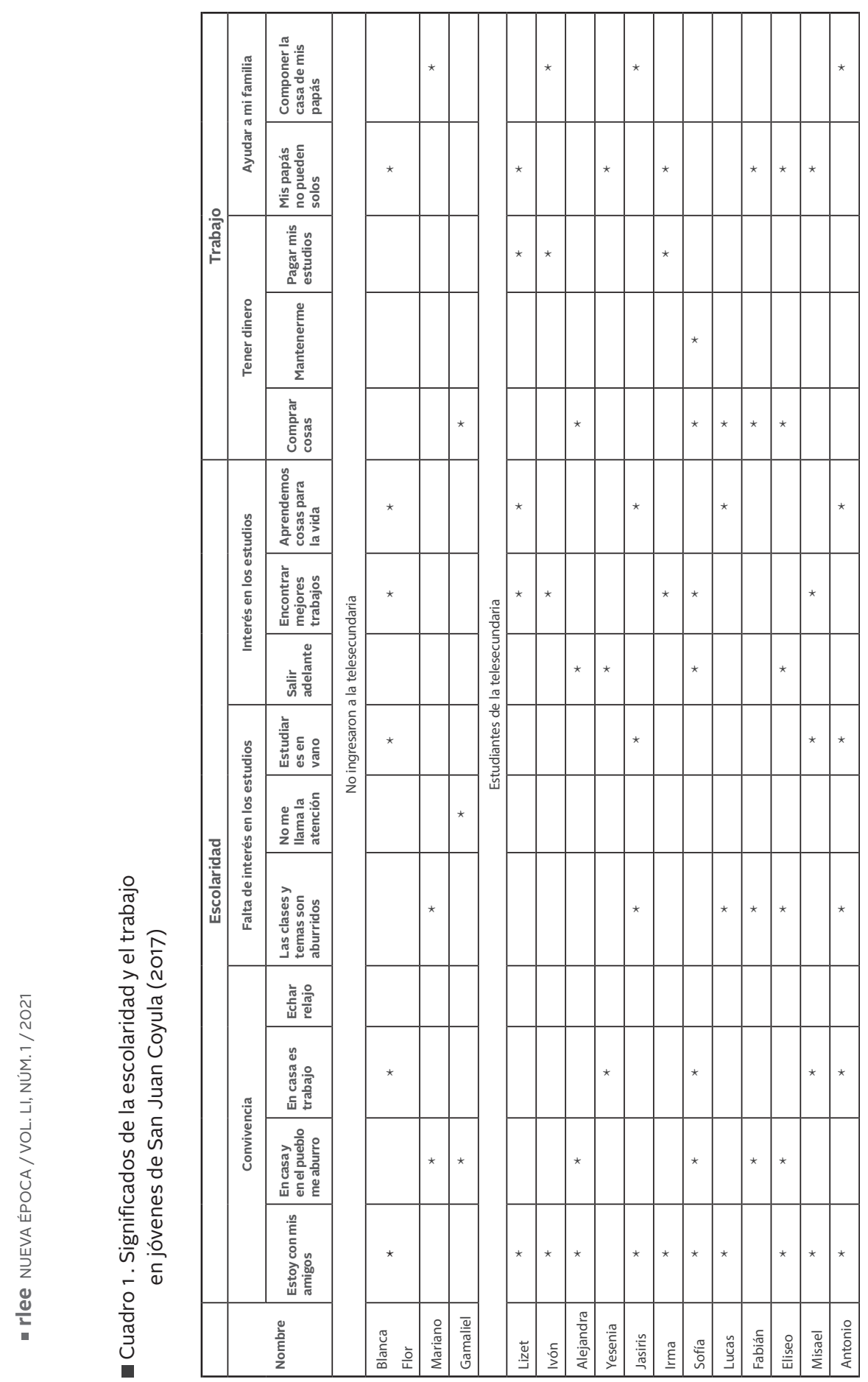



Blanca Flor (E25-08-2017, no ingresó a la telesecundaria) explica que quisiera regresar a la escuela, ya que "en mi casa sólo me ponen a hacer quehacer. Cuando iba a la primaria no se me exigía tanto, me distraía y divertía. Pero como ahora no voy, no puedo hacerlo". Blanca Flor compara su vida de estudiante y la de ahora. Su asistencia a la escuela le permitía distraerse y divertirse, pero ahora "como yo soy la que está todo el día con mi mamá, ella me manda a comprar, a barrer o a llevarle taco a mi papá al campo". A Blanca Flor le gustaría regresar a la escuela, para poner un paréntesis a las demandas de la casa.

Otro caso es el de Misael (E03-04-2017, alumno de $3^{\circ}$ de telesecundaria), quien expresa que "en las tardes me dedico a trabajar y no puedo ver a mis amigos. A veces me los encuentro en la moto, pero no me quedo a platicar con ellos porque mi mamá se enoja”. Para Misael, la escuela es un lugar en el que no sólo puede convivir, también es un lugar en el que toma un descanso de su jornada de trabajo, pues "llego a mi casa, mi mamá me da de comer y de ahí agarro el mototaxi para trabajar y ya regreso como a las nueve [de la noche] y ya si me da ganas hago la tarea, si no, ya la dejo". En clase frecuentemente se observa a Misael con sueño o cansado, por lo que a veces se recuesta en el asiento para descansar, a tal grado que le agarra el sueño; en una ocasión, la profesora le cuestionó el porqué de su cansancio y respondió: "es que ayer trabajé hasta tarde y llegué bien noche a mi casa” (O22-03-2017).

La participación de los jóvenes coyuleros en quehaceres domésticos o actividades agrarias llevan a que la escuela funja como una especie de escape para reunirse, pero sobre todo para descansar.

\section{Echar relajo}

La escuela como espacio en el que pueden echar relajo va más allá del simple disfrute de estar juntos. El echar relajo es mencionado por los jóvenes coyuleros como la trasgresión del orden. Karla (E29-06-2017, egresada de la telesecundaria) indica que "no entraba a clases. Sólo iba a la escuela para echar relajo con mis amigos". Su bajo desempeño escolar y los reportes hicieron que sus padres la sacaran del bachillerato.

El caso de Aldair (E14-06-2017, abandonó el bachillerato) se parece al de Karla. Él comenta que cuando asistía al bachillerato de la 
comunidad vecina, sus padres no lo vigilaban y podía echar relajo; "iba a cursar mi tercer semestre [de bachillerato], empecé a echar relajo en la escuela, a faltar y a consumir drogas con unos amigos. Entonces, mi mamá lo llegó a saber y me dio de baja”.

Algunos padres de familia están contra de que sus hijos asistan a la escuela, ya que nada más van a echar relajo, sobre todo si se trata de enviarlos fuera de la comunidad. Por ejemplo, Doña Lucía expresa que "a Cuyamecalco [comunidad vecina] ya no tuve la confianza de mandarla, porque ya no los ve uno, ya no se sabe qué están haciendo" (E25-08-2017, mamá de Jasiris). Por su parte, Don Edipo (E25-08-2017, papá de Jasiris) menciona su preocupación: "de que se van solos, quién los vigila, quién les dice algo".

Lo anterior ilustra que para las familias coyuleras la estrategia de enviar a los jóvenes a estudiar fuera de la comunidad no está funcionando. Lejos de la vigilancia de los padres, los jóvenes empiezan a orientarse por el relajo, a juntarse con amigos para faltar a clases y a dejar de lado su formación escolar.

\section{Falta de interés en los estudios}

\section{Las clases y temas son aburridos}

Para algunos jóvenes los temas son aburridos porque piensan que ya han aprendido lo suficiente para trabajar. Jasiris (E04-04-2017, alumna de $3^{\circ}$ de telesecundaria) menciona que le interesa "aprender, por ejemplo, a hacer las cuentas, porque, tal vez más tarde encontremos un trabajo y así ya podríamos facilitarnos más en hacer las cuentas para trabajar". Jasiris tiene la expectativa de trabajar atendiendo una tienda de ropa o de empleada doméstica. Sus hermanos le comentan que al terminar la secundaria se vaya a la Ciudad de México; "mi hermana me dijo que me anda consiguiendo un trabajo, para que me vaya con ella”. Avergonzada ańade, “a veces sí, de plano me entra la flojera en no poner atención”. Y es que en clases frecuentemente desatiende las explicaciones de la profesora o deja de lado las actividades académicas: "mientras algunos trabajan en la actividad propuesta por la maestra, Jasiris mira a la ventana y se entretiene mirando a su alrededor; voltea al salón, observa sus libretas y bosteza" (O23-03-2017). 
La mayoría de los jóvenes coyuleros tiene la expectativa de migrar a la ciudad para trabajar, en el caso de las mujeres como empleadas domésticas o atendiendo una tienda de ropa y en el de los hombres, como vendedores ambulantes, choferes o albañiles. Para cumplir dichas expectativas, es suficiente con saber leer y hacer cuentas básicas.

En otros casos, como el de Karla y Eliseo, el aburrimiento de los temas no sólo proviene de sus expectativas, sino de la forma de enseñanza de los profesores. Karla indica que "tenía un profesor que sólo se la pasaba explique y explique los temas, pero su explicación era demasiado aburrida”. Luego "en la telesecundaria, recuerdo que a veces nos dejaban trabajos y se salían a la dirección; nosotros mejor nos poníamos a jugar”.

En una observación en la telesecundaria conversé con Eliseo; de manera espontánea, le pregunté si seguiría estudiando, a lo que él contestó: "no sé, en veces sí y en veces no. Es que me da flojera" (O22-03-2017). El "en veces si”" que menciona se justifica de lo que él cree el deber ser, "si estudiamos, podemos alcanzar una carrera y eso nos ayuda a salir adelante; pa'tener un trabajo más o menos". En el caso del "en veces no" que comparte, tiene que ver con la flojera provocada por su falta de interés en los estudios, que se origina a partir de que observa que las clases son aburridas; "los trabajos luego son aburridos, nada más estar escribiendo o nos dicen que hagamos una lección y ya con eso". En una ocasión, cuando todos salieron del salón, me acerqué a su lugar, en su asiento se encontraba escrito: "nadie entiende a los maestros" (O30-03-2017).

Algunos jóvenes justifican que el aburrimiento que expresan hacia los temas escolares proviene de las estrategias pedagógicas implementadas por sus profesores.

\section{No me llama la atención}

Algunos jóvenes coyuleros sienten que la escuela no les permite desarrollar sus habilidades. Jorjiet (E24-04-2017, egresada de la telesecundaria) menciona que, desde pequeña, ella junto con sus amigas querían ser estilistas profesionales, "nos habíamos hecho a la idea de estudiar cultura de belleza, porque nos llama mucho la atención la cuestión de peinados y maquillaje". Comenta que la escuela no le llama la atención, ya que "creo que lo mío es cultura de belleza, 
la escuela sí, pero me gusta ser estilista”. Ella espera que su padre se recupere pronto para que pueda regresar a la Ciudad de México e iniciar nuevamente sus cursos.

El caso de Héctor (E17-06-2017, abandonó la telesecundaria en $2^{\circ}$ ) es similar al de Jorjiet: en su estancia en la escuela siempre se dedicó a echar relajo, hasta que se dio cuenta que la escuela "no me llamaba la atención”. Héctor empezó por faltar hasta que dejó de ir completamente a clases, de acuerdo con él, su interés reside en aprender mecánica, “a mí lo que me gusta es la mecánica, componer carros y eso, pero aquí no hay donde. Aunque se lleven matemáticas, ahí creo que sí me gustarían”.

La experiencia de Héctor es semejante a la de Gamaliel (E05-072017, no ingresó a la telesecundaria), quien comenta que desde que iba a la primaria no le gustaba la escuela: "no me llamaba la atención, porque no le encontraba sentido”. Él menciona que la única clase que le gustaba era la de formación cívica, ya que ahí “a veces hacíamos esculturas". Lo anterior me parece relevante, ya que Gamaliel ganó en la primaria un concurso de dibujo y le gusta tallar figuras en madera. Gamaliel comparte que hacer una figura de madera le lleva alrededor de dos o tres semanas, ya que primero debe ir al campo a buscar ramas de árboles que tengan ciertas figuras; "hice un chango y un hombrecito, simulando que se estaban cargando. Esas figuras las consigo en mi corral, porque ahí hay espina y de esos árboles salen mejor”. Como se puede ver, Gamaliel tiene habilidades no académicas, por lo que va a la escuela sin ganas.

Los casos de Jorjiet, Héctor y Gamaliel muestran una especie de crítica a la escolarización; tienen intereses en una formación más práctica y menos académica, como el aprendizaje de un oficio. Los jóvenes que mantienen este significado asisten a la escuela sin ganas, por lo general van a echar relajo; muchas veces son tildados como malos estudiantes. La pobreza económica de las familias y de la comunidad en general cobra relevancia para que los jóvenes no puedan orientarse a desarrollar sus intereses. En este contexto, aunque la escuela es un espacio relevante para la convivencia con otros jóvenes, sus principales intereses no están ahí.

Al contrastar las expresiones de este grupo de jóvenes con aquellos que mencionan que en la escuela aprendemos cosas para la vida (véase más adelante) sobresale otra interpretación: la escuela no es 
relevante para algunos jóvenes coyuleros, porque piensan que la experiencia en ella no repercute en su vida cotidiana e intereses futuros.

\section{Estudiar es en vano}

Erick y Luis Miguel comparten que la escolaridad no te permite encontrar mejores trabajos. Para comprender este planteo, partamos de los razonamientos de Erick.

Erick (E26-06-2017, egresado de la telesecundaria) considera que terminar estudios de bachillerato no tiene sentido, ya que al final va a terminar trabajando de albañil. Agrega que los estudios implican gastos económicos que su familia no puede costear: "uno de mis primos terminó el bachillerato; él quería ir a la universidad, pero sus papás ya no lo pudieron apoyar. Ahora él trabaja en la albañilería”. Entonces, el esfuerzo de terminar el bachillerato no habrá valido la pena; por el contrario, estudiar el bachillerato le resta tiempo para avanzar y adquirir experiencia en la albañilería.

Lo que comenta Luis Miguel complementa lo compartido por Erick; él menciona otro elemento, mediante la escolaridad es posible encontrar un trabajo físicamente menos demandante, pero "luego no los pagan bien [...] uno de mis amigos que terminó el bachillerato me dijo que entró a trabajar en una tienda en la que le pagaban $\$ 4000$ pesos mensuales, por lo que mejor se metió a la albañilería. Aquí uno puede sacar los $\$ 5000$ o $\$ 6000$ pesos al mes". En este contexto, Erick y Luis Miguel prefieren ingresar a la albañilería.

Los razonamientos de Erick y Luis Miguel también fueron expresados por algunos padres de familia que se encuentran contra de la escolarización de sus hijos. Don Misael (E24-08-2017, papá de Misael) piensa que "el estudio no vale, porque a veces, estudian y no hay trabajo. Por ejemplo, mi hermano se esforzó estudiando y terminó vendiendo en un negocio". Don Misael duda de la correlación entre la escolaridad y el trabajo, por lo que expresa no apoyar a su hijo. En Coyula hay personas que con una formación superior no tienen trabajo; estas experiencias llevan a que la mayoría de las familias coyuleras vean con duda la escolarización de los jóvenes (N20-06-17).

Para algunas familias rurales, la devaluación de las credenciales escolares (De Ibarrola, 2016) y el déficit de demanda laboral cualifi- 
cada se complementan y ponen en duda la rentabilidad económica de la escolaridad (Muñoz, 2006).

Lo anterior refuerza el razonamiento de que para trabajar no se necesita estudiar expresado por seis de los ocho padres entrevistados. Doña Isabel (E22-08-2017, mamá de Daniela) comparte: “mis papás decían que para echar tortillas no se estudia”. Por su parte, Don Edipo menciona: "crecimos trabajando en el campo. Mi papá decía que debíamos aprender a trabajar para vivir, que a la escuela sólo íbamos a pasar el rato".

Los escasos efectos (económicos) que observan las familias rurales de la escolarización refuerza la razón heredada por sus predecesores de que para trabajar no se necesita estudiar, por lo que la mayoría de los padres se muestra contra la escolarización de sus hijos.

\section{Interés en los estudios}

\section{Salir adelante}

En este contexto, es relevante analizar los razonamientos de aquellos jóvenes que tienen interés en la escolaridad pues, ¿qué aspectos encontramos en sus expresiones y experiencias de vida que los lleven a interesarse en la escolaridad?

El salir adelante, que menciona Norma (E03-07-2017, estudiante de bachillerato), tiene que ver con la comparación que hace de su vida y la de sus padres, ya que [la escolaridad] "ayuda a tener una vida mejor; no como la de nuestros padres". De acuerdo con su comparación, la vida de sus padres está llena de dificultades debido a su escasa escolaridad; sus padres le dicen que: "debo estudiar, porque si no, me va a costar mucho. Mi mamá luego me dice: 'ya ves a nosotros, luego nos hacen tontos en las cuentas"”.

Se puede observar que para algunos jóvenes coyuleros el interés de adquirir mayor escolaridad es que ésta les permitirá una vida mejor que la de generaciones precedentes. Otro caso parecido es el de Yesenia, quien comparte su interés de alcanzar mayor escolaridad, ya que "si estudiamos podemos ayudar a cuidar nuestro pueblo, a ver que se hagan cosas aquí”. La idea de mayor escolaridad en ella tiene que ver con un sentimiento colectivo de ayudar a mi comunidad mencionado por algunos jóvenes rurales e indígenas (Pacheco, 2016). 
En contraste con Yesenia, encontré a Alejandra (E05-04-2017, alumna de $3^{\circ}$ de telesecundaria); ella se ha apropiado de los consejos de sus padres: "mis papás me dicen que para qué me voy a quedar en el pueblo, como muchas de las mujeres que se quedan en la casa a barrer y moler, lo cotidiano [labores domésticas]. Ellos no quieren eso para mí”. En algunos jóvenes la idea de adquirir mayor escolaridad funge como un medio que les permite migrar de sus comunidades y ampliar su horizonte de posibilidades.

Se observó que algunas mujeres expresan un fuerte interés por adquirir la mayor escolaridad posible, y es que las jóvenes se encuentran construyendo proyectos en los que la escolaridad ocupa un lugar importante; ellas se encuentran aplazando la idea de casarse, porque primero termino una carrera y empiezo a trabajar. De acuerdo con sus observaciones y los consejos de sus madres, estas mujeres van construyendo la expectativa de que la escolaridad les permita salir del encierro doméstico (en condición de amas de casa) e ingresar a trabajos para compartir gastos con su esposo, con la intención de que no falten recursos económicos en sus familias de procreación. Pero también para adquirir autonomía e independencia (Tapia, 2015).

Algo que llamó la atención es que la mayoría de los varones no mostró interés en la escolaridad, ¿qué pasa con ellos? (N28-06-17). Sofía (E04-07-2017, alumna de $3^{\circ}$ de telesecundaria) respondió: "la mayoría de los hombres tiene que trabajar para mantener a la familia. Según los padres, de que terminas, se van a trabajar y dejan de estudiar, eso tiene que ver con mis compañeros". Sofía hace alusión a que, en el caso de los hombres, éstos asumen la responsabilidad de ayudar en el gasto familiar, por lo que la escolaridad queda como un elemento secundario.

Las mujeres interesadas en obtener mayor escolaridad desarrollan estrategias. Por ejemplo, Norma indica que, por las tardes, mientras atiende el negocio familiar, realiza las tareas de la escuela. Por su parte, Yesenia compartió que llega a su casa para ayudar a su mamá a hacer la comida, después se apura a ayudar a hacer la limpieza, "termino lo más rápido posible para empezar a hacer mi tarea”.

Para los hombres es complicado desarrollar estrategias, debido a que después de la escuela realizan trabajos demandantes. Por ejemplo, Antonio (E15-06-2017, alumno de $3^{\circ}$ de telesecundaria) menciona que después de la escuela llega a su casa a comer para llevar a los chivos a pastar al campo o ir a limpiar la siembra de temporal. 
Ahora bien, Misael comenta que después de las clases se pone a trabajar en el mototaxi y llega a casa a las diez de la noche. Antonio y Misael llegan cansados a sus casas, por lo que no realizan tareas escolares y en la clase se muestran cansados.

\section{Encontrar mejores trabajos}

Se puede observar que la escolaridad permite salir de los trabajos tradicionales (ama de casa o empleada doméstica).

Blanca Flor explica que la escuela le gusta porque quiere "conocer más para un mejor trabajo". Ella opina que los estudios le permiten realizar una profesión; en el fondo, busca dejar de lado la labor de ama de casa.

Por su parte, Lizet (E25-06-2017, alumna de $3^{\circ}$ de telesecundaria) tiene claro que necesita una mayor escolaridad para encontrar un mejor trabajo; ella se apropió de este significado a través de los consejos de su tía; "ella me dice que necesito el bachillerato para trabajar en alguna tienda grande y no sólo de empleada doméstica".

Algunos padres comparten con sus hijos la importancia de la escolaridad, porque les va a hacer falta para trabajar. Doña Zobeida (E24-08-2017, mamá de Misael) comenta que "ahora para todo piden papeles [certificado]. Si quieres ir a la ciudad, se necesita llevar por lo menos el bachillerato". Estos razonamientos provienen de su experiencia en la ciudad realizando trabajos demandantes físicamente; por ejemplo, Don Pedro (E30-08-2017, papá de Alejandra) comparte que, cuando estuvo en Estados Unidos trabajando de albañil: "esa no era vida. Pero aguanté diez años. Eso me fue motivando para que a mi hijo no se le metiera esa idea de no estudiar".

Por otro lado, el interés de la escolaridad para encontrar mejores trabajos figuró en las expresiones de algunos jóvenes que abandonaron la escuela.

Daniela (E03-07-2017, abandonó la telesecundaria) comparte que ha trabajado como empleada doméstica y como mesera. Según ella "cuando trabajé de empleada doméstica tenía que limpiar toda la casa, hacer la comida y luego, como la señora tenía un niño de tres años, lo tenía que cuidar". Debido a la exigencia del trabajo, decidió buscar otras oportunidades. Daniela consiguió empleo en un restaurante, ella tenía que llegar a las siete de la mańana para limpiar el lugar; asimismo, debía lavar trastes y atender a la cliente- 
la. La salida del restaurante era a las ocho de la noche, pero a veces se extendía hasta las diez, lo que hacía que llegara a media noche al cuarto que rentaba. Daniela indica que esto no le gustó para nada, por lo que buscó un trabajo de empleada en un centro comercial, específicamente en una tienda departamental; la respuesta que recibió es que necesitaba el certificado de bachillerato.

Por su parte, Abdiel (E14-06-2017, abandonó el bachillerato) comenta: "me fui a trabajar y se me hizo fácil, pero como, la verdad, yo no estaba acostumbrado a trabajar, sí se me hizo muy pesado; ya con trabajo tenía que levantarme temprano, cargar botes de cemento". Abdiel pensaba que como albañil iba a vivir como siempre había querido; sin órdenes ni amenazas, libre y despreocupado.

Daniela y Abdiel reflexionan acerca de la importancia de la escolaridad para encontrar mejores trabajos; su experiencia laboral configuró en ellos una especie de punto de retorno que "los llevó a replantearse los estudios y asumirlos con una nueva actitud" (Guerrero, 2012, p. 137).

\section{Aprendemos cosas para la vida}

Algunos jóvenes señalaron que en la escuela les enseñan cosas que no han aprendido en casa. Jasiris menciona que le gusta la clase de formación cívica y ética, ya que abordan temas "sobre qué debe hacer uno si tenemos problemas, eso sí me parece muy interesante”. Jasiris alude específicamente a las dudas que le surgen sobre el comportamiento; la profesora les aconseja en clase que deben darse a respetar, que ambos géneros son iguales. Jasiris comparte que en particular le gusta cuando abordan temas de sexualidad, ya que ha aprendido "que debemos tener cuidado si ya tenemos novio, porque si no nos cuidamos, podemos quedar embarazadas".

Por otro lado, Antonio indica que "nos enseñan cosas que no hemos aprendido y que son importantes para nuestra vida". Al preguntarle qué cosas son importantes para su vida, él responde: "los temas de formación... ya que nos enseñan a saber más de cómo organizarnos en el pueblo".

Los temas escolares que a Jasiris y a Antonio les despiertan interés son aquellos que toman en cuenta su vida cotidiana (dudas personales, expectativas laborales, organización política de su comunidad). 


\section{SIGNIFICADOS Y SENTIDOS DEL TRABAJO}

\section{Tener dinero}

\section{Comprar cosas}

Una de las principales coincidencias entre Alejandra y Abdiel es que tienen la idea de trabajar para comprarse cosas; Alejandra expresa que su idea de trabajar es "para tener dinero y comprarme mi computadora o un celular". Y es que a sus papás apenas y les alcanza para pagar los estudios de sus tres hijos.

Por su parte, Abdiel indica que trabajar le otorga la posibilidad de comprarse ropa: "cuando estaba estudiando, solamente tenía cuatro mudas de ropa. Ahora, con el dinero que gano, me voy comprando mis cosas; apenas compré un pantalón y dos pares de zapatos”. Esto lo ha llevado a dudar si quiere regresar a la escuela; él duda que pueda acostumbrarse a no traer dinero y dejar los gustos que se ha dado desde que trabaja.

La idea de trabajar para comprar cosas se presenta desde muy pequeños. Dońa Isabel expresa: "mi hijo que va a salir de la primaria me dice que ahora que salga ya no va a ir a la secundaria, se quiere poner a trabajar para comprar su celular, porque ve que sus compañeritos tienen celular".

El motivo de trabajar para comprar cosas que mencionan los jóvenes coyuleros proviene, principalmente, de las carencias económicas en las que viven.

\section{Mantenerme}

De acuerdo con Sofía y Luis Miguel, el trabajo les permite obtener dinero para lograr cierta independencia económica de su familia.

Sofía expresa dos significados fuertes del trabajo: piensa que es importante porque "ayuda a que uno se mantenga, a que dejemos un poco a nuestros padres y nos hagamos cargo de nosotros mismos”. La idea de trabajo en Sofía radica en adquirir cierta independencia; comenta que, cuando trabaje, tratará de no causarles molestias económicas a sus padres, "está mal eso de que uno esté toda la vida con los papás; creo que debemos trabajar y empezar a mantenernos". 
El caso de Luis Miguel es trágico, ya que sus padres murieron cuando él tenía seis años, por lo que quedó al cuidado de sus hermanos mayores; ellos lo ayudaron a que terminara la primaria y la secundaria, por lo que su idea del trabajo es que le ayuda a mantenerse.

En las conversaciones con estos jóvenes se puede analizar que el significado de trabajar para mantenerse cobra dos sentidos relevantes: conseguir cierta independencia familiar y debido a problemas familiares.

\section{Pagar mis estudios}

Este significado fue mencionado sólo por las mujeres que se encuentran interesadas en adquirir mayor escolaridad. Lizet comparte que el trabajo le va a permitir tener dinero para pagar sus estudios. Lizet es consciente de que "es mucho gasto para ellos [sus padres], yo siento que no pueden". Sus padres comparten con ella la situación económica familiar: "ellos me dicen que no tienen mucho dinero, como tengo hermanos pequeńos; luego se enferman o también van a la escuela y es mucho gasto".

Los razonamientos de Ivón (E23-06-2017, alumna de $3^{\circ}$ de telesecundaria) e Irma (hermanas) son parecidos a los de Lizet. Sólo que ellas piensan combinar el trabajo con la escuela, ya que su padre les ha comentado que sólo las apoyará a que terminen el bachillerato en la comunidad vecina.

Para la mayoría de jóvenes coyuleros es secundario trabajar para pagar sus estudios, ya que están dispuestos a dejar la escuela si surge un imprevisto familiar.

\section{Ayudar a mi familia}

\section{Mis papás no pueden solos}

En los casos de Blanca Flor y Misael su posición de hijos mayores va configurando el sentido de responsabilidad de ayudar a sus padres a trabajar y colaborar en el gasto familiar, por lo que sacrifican -contra de su voluntad-intereses individuales.

Blanca Flor es la hija mayor; ella siente la responsabilidad de ayudar a sus padres: "yo me oriento en trabajar, porque quiero ayudar a mis papás, ellos no pueden solos con mis hermanitos y debo 
echarles la mano", por lo que relega la idea de estudiar para ponerse a trabajar y ayudar en el gasto familiar.

Para Misael el trabajo es algo cotidiano. De acuerdo con él, "tengo que ayudar a mi papá para que mantengamos la casa”. La precariedad económica de la familia hace que los jóvenes como Misael contribuyan cotidianamente de diversas maneras en la reproducción familiar, esto va configurando una especie de deuda familiar.

La edad de los padres cumple un papel importante. Por ejemplo, el padre de Jorjiet tiene 60 ańos, ya no puede dedicarse al campo; "mi papá ya no puede trabajar y mi mamá es ama de casa, por eso me preocupan ellos $y$, cuando puedo, les mando dinero, sobre todo si necesitan medicamentos". El interés de Jorjiet por ayudar a sus padres ha hecho que deje de lado su objetivo de estudiar cultura de belleza.

Cabe señalar que en Coyula, trabajar para ayudar a la reproducción familiar es una cuestión cultural. Los padres mencionaron que, al igual que sus hijos, cuando eran jóvenes participaban en la reproducción familiar; esto se ejemplifica con lo que comparte Doña Alicia (E30-08-2017, mamá de Alejandra), "nosotros muy jóvenes salimos del pueblo para trabajar. Tenía la responsabilidad de apoyar para el gasto de la familia". Según Dońa Alicia, "nuestros papás nos dieron esa responsabilidad de trabajar y no la de estudiar". Actualmente algunas familias reflexionan ante este significado heredado. Por ejemplo, Dońa Alicia indica que no es responsabilidad de los hijos trabajar y ayudar en el gasto familiar. Sin embargo, la mayoría de los padres implícita o explícitamente evidencian que este significado sigue vigente. Dońa Zobeida indica que su esposo (Don Misael) "es de otra mentalidad, como ya van creciendo los nińos, dice que ya deben trabajar".

Lo anterior lleva a afirmar que, en Coyula, ayudar a los padres en la reproducción familiar es una cuestión cultural vigente.

\section{Componer la casa de mis papás}

Jasiris menciona que el trabajo les proporciona el dinero para componer la casa de sus padres. Jasiris señala que sus padres le han comentado que le van a heredar una parte del terreno familiar. Entonces, ella piensa construir ahí su casa, y para ello tiene que trabajar. 
La mayoría de jóvenes vive en casas de adobe con techo de palma, por lo que buscan construir casas de varilla y cemento (N22-03-2017). Por ejemplo, los hermanos de Jorjiet y Fabián buscan construir casas semejantes a las de Estados Unidos o parecidas a las mansiones donde trabajan de jardineros, albañiles o empleadas domésticas.

El significado de componer la casa de los padres es expresado por algunos jóvenes coyuleros porque heredarán la casa, porque éstas son pequeñas o porque buscan construir una casa de material.

\section{CONCLUSIONES}

\section{La convivencia y la falta de interés en los estudios como las categorías más importantes}

Todos los jóvenes rurales que se entrevistaron mencionan que la convivencia es el significado más importante por el que asisten a la escuela. Como se describió anteriormente, este significado proviene de los escasos lugares en los que se pueden reunir con sus amigos. En específico, a las mujeres les permite salir del encierro doméstico (en condición de amas de casa) que aún sigue vigente en muchas comunidades rurales e indígenas. Además, la escuela es un espacio de descanso ante las tareas agrarias. Para la mayoría de los jóvenes, la obtención del certificado es secundario. Esto se debe a que sus expectativas son culminar la telesecundaria e irse a la ciudad a trabajar como empleadas domésticas o atendiendo una tienda en el caso de las mujeres, o como campesinos o albañiles en el caso de los hombres. Para ellos la escuela funge como un paréntesis en sus proyectos laborales u otros intereses que están fuera de sus muros (Reyes, 2010). Este hallazgo se contrasta con lo encontrado por Guerra y Guerrero (2004), quienes observaron que para los jóvenes urbanos el motivo principal para asistir a la escuela es la obtención del certificado para cursar estudios del siguiente nivel.

La convivencia se combina con la falta de interés en los estudios que muestran y expresan la mayoría de los jóvenes. Los estudiantes comparten que las clases y temas son aburridos. Este significado evidencia "una crítica extendida por parte de los estudiantes a las prácticas de enseñanza que desarrollan los profesores" (Reyes, 2014, p. 
91). Cabe resaltar que algunos estudiantes indicaron que los temas de formación cívica y ética son interesantes porque son explicados considerando aspectos de su vida. Por su parte, los que abandonaron la escuela o que ya no siguieron estudiando mencionan que la escuela no me llama la atención o que estudiar es en vano, esto permite reflexionar en las estrategias pedagógicas; se propone apasionar a los jóvenes mediando el horizonte académico con situaciones más significativas en términos de vida y experiencia juvenil (Weiss, 2018).

Por otro lado, algunos jóvenes establecen una crítica a la idea de escolarizar sus vidas, ya que mencionan que la escuela no me llama la atención; este grupo muestra que hay jóvenes que tienen intereses, capacidades o habilidades más allá de adquirir una formación académica (Auli, 2019). En estos casos, es posible observar la manera en que -a veces- la escolarización oscurece las habilidades o capacidades de las personas (Gimeno, 2000). Los intereses de la mayoría de los jóvenes rurales provienen de la expectativa de egresar de la telesecundaria y ponerse a trabajar, pero también a partir de inclinaciones subjetivas. $\mathrm{Al}$ respecto, sería importante que el sistema educativo amplíe alternativas educativas. Por ejemplo, aquellas encaminadas a la formación para el trabajo, que faciliten la inserción laboral de aquellos jóvenes que no tienen el objetivo de conseguir una profesión. Complementariamente, habría que contemplar (o reforzar) en las escuelas pláticas de orientación vocacional que ayuden a los jóvenes a explorar sus habilidades y capacidades.

La falta de interés en los estudios también surge de percibir que estudiar es en vano; este significado enriquece la postura disfuncional entre escolaridad y trabajo: la escolaridad no produce mejores posiciones laborales o mejora los ingresos económicos. En este sentido, para las familias rurales adquirir mayor escolaridad no es suficiente para lograr movilidad social.

En general, la falta de interés en los estudios que muestran la mayoría de los jóvenes coyuleros confirma lo observado en múltiples jóvenes provenientes de familias obreras o rurales: el desencanto escolar proviene de la resistencia ante la imposición de la hegemonía cultural, de la oposición a las reglas de los adultos o de las expectativas de los jóvenes (Willis, 1988; Feito, 1990; Reyes, 2010; Fernández, 2011; Miranda, 2012). 
La convivencia y la falta de interés en los estudios se complementan haciendo que algunos jóvenes rurales empiecen a ausentarse de la escuela hasta abandonarla.

Cabe resaltar que, de los 26 jóvenes entrevistados, sólo ocho mujeres y tres varones expresan un interés en los estudios. Según sus razonamientos, la escolaridad les permite salir adelante y encontrar mejores trabajos. Los consejos de la familia son importantes; en algunos casos la experiencia laboral en un trabajo demandante (físicamente) los ha llevado a un punto de retorno y a plantearse la idea de regresar a estudiar para encontrar mejores trabajos.

La precariedad de las comunidades rurales no ofrece alternativas para los jóvenes que abandonan la escuela, por lo que es posible observar a muchos realizando mandados, usando el celular y viendo televisión todo el día; en el caso específico de los varones, éstos se juntan para jugar futbol o platicar. Esporádicamente realizan trabajos agrarios. Las condiciones en las que viven estos jóvenes rurales son parecidas a las de los ninis (ni estudian ni trabajan) urbanos. Es preciso aclarar que su condición de ninis no procede de una elección deliberada, sino de la falta de oportunidades que su contexto ofrece. Esta situación es preocupante, ya que la pobreza, los intereses de los jóvenes por ocuparse y la poca orientación de los padres, los pueden conducir a la delincuencia.

\section{Trabajar es más importante que asistir a la escuela}

Algunos jóvenes coyuleros mencionan la idea de trabajar para tener dinero; este significado proviene, principalmente, de las carencias económicas en las que viven. Además, el trabajar para comprar cosas expresado por algunos jóvenes rurales deriva de la creciente expansión de la sociedad de consumo (Bourdieu y Sayad, 2017), ya que se alejan de sus predecesores, que trabajaban principalmente para subsistir. Actualmente, los jóvenes rurales también buscan trabajar para comprarse ropa, zapatos, un celular y otros productos. Es posible que lo anterior implante en las comunidades rurales una percepción del trabajo egoísta-individualista, dejando de lado las concepciones tradicionales que -como se describe enseguida- siguen vigentes.

Los jóvenes rurales participan cotidianamente en la reproducción familiar, por lo que van configurando el significado sobre el 
trabajo como medio para ayudar a mi familia. El conocimiento de los jóvenes acerca de la económica precaria de su familia les genera la deuda de trabajar para apoyar a sus padres. En este sentido, el significado concreto de trabajar porque mis papás no pueden solos proviene de la responsabilidad, preocupación y deuda familiar que sienten, por lo que la mayoría está dispuesta a renunciar a objetivos individuales y ponerse a trabajar para ayudar a la familia (Langa y Río, 2013; Tapia, 2015).

Trabajar para ayudar a la familia es una característica cultural vigente en las comunidades rurales e indígenas, ya que como cuentan los padres -al igual que sus hijos- en su momento asumieron la responsabilidad y deuda de trabajar para contribuir en el gasto familiar, esto se encuentra dentro de un sentido de "reciprocidad familiar" (Tapia y Weiss, 2013).

La dimensión cultural cobra un peso fundamental en los significados que los jóvenes rurales otorgan a la escolaridad y al trabajo. En este contexto, trabajar es más importante que estudiar.

\section{La dominación femenina de la escolaridad}

La escolaridad ofrece a las mujeres rurales construir expectativas y aspiraciones diferentes de las tradicionales, como amas de casa o empleadas domésticas en la ciudad; les permite superar la condición de género que ocupan en sus familias y comunidad. También les ayuda a adquirir autonomía e independencia (Tapia, 2015, 2019).

Las mujeres interesadas en obtener mayor escolaridad desarrollan estrategias como terminar sus actividades en el hogar (hacer la comida, lavar la ropa y limpiar la casa) con la intención de abrir un espacio que les permita realizar sus tareas escolares. En este contexto, es más probable el éxito escolar de las mujeres (Gómez et al., 2001).

Por su parte, los hombres tienen la responsabilidad de irse al campo a cuidar el ganado, sembrar o cosechar, por lo que llegan cansados a sus casas o cuentan con escasos espacios para realizar las tareas escolares. En este contexto, es poco probable el éxito escolar de los varones.

Los significados y expectativas de estas mujeres se complementan con la coyuntura crítica que vive el campo. Debido a la carestía del campo, las familias empiezan a hacer frente a las condiciones cambiantes del entorno, por lo que realizan proyectos familiares en 
los que fomentan la permanencia de las mujeres en la escuela, mientras que los varones se insertan más temprano al trabajo (Estrada, 2007, Tapia, 2015).

Es posible afirmar que en las comunidades rurales existe una dominación femenina de la escolaridad. Las mujeres se encuentran significando de manera positiva la adquisición de mayor escolaridad. Por su parte, son contados los hombres que muestran interés en los estudios; esto se debe al rol tradicional que ocupan en la comunidad. Los varones cuentan con más derechos que las mujeres en la toma de decisiones familiares y comunitarias, pero también tienen más responsabilidades; los hombres (sin importar su condición de padres o hijos) son los principales proveedores de recursos económicos, no sólo por cuestión cultural, sino porque la precariedad económica de la mayoría de las familias rurales así lo exige.

\section{REFLEXIONES GENERALES}

La presente investigación confirma lo señalado por Silva y Weiss (2018) acerca de que los problemas familiares son una razón importante para que los jóvenes muestren falta de interés en los estudios; en las comunidades rurales, la migración de los padres a la ciudad genera desintegración familiar. Asimismo, la muerte o enfermedad de algún miembro de la familia se convierte en un imprevisto que impide que los padres apoyen la escolaridad de los jóvenes. En estas condiciones, los jóvenes se encuentran más susceptibles a abandonar la escuela. Por lo anterior, tal vez sea necesario que los profesores impartan talleres en los que participen los jóvenes y sus familias para desarrollar la integración familiar, combinadas con visitas domiciliarias (como en algún tiempo se hacía) con la intención de concientizar a los padres de la importancia de la escolaridad; ésta no como medio para alcanzar progreso económico, sino como la oportunidad para que los jóvenes se acerquen al conocimiento.

Persiste la idea de que la razón económica es la principal para que los jóvenes y sus familias muestren falta de interés en la escolaridad. En el presente estudio no fue una razón importante, tal vez porque la mayoría de jóvenes entrevistados son estudiantes de telesecundaria, escuela que se encuentra en la comunidad y que les proporciona una 
beca, también puede deberse a que los egresados de la telesecundaria ya no siguieron estudiando. Cabe señalar que los jóvenes que aludieron a cuestiones económicas son aquellos que estudian o abandonaron el bachillerato, ellos comentan que la beca que reciben en el bachillerato no es suficiente, ya que gastan en traslados a la cabecera municipal, lugar en el que se encuentra el bachillerato, a lo que se agregan los gastos de material escolar.

Los resultados aquí expuestos agregan elementos para considerar alternativas educativas y de atención a los jóvenes rurales con el objetivo que el mayor número de jóvenes acceda a la educación obligatoria. Se propone que, además de otorgar becas a los estudiantes, construir y ampliar escuelas, también se ofrezca un servicio educativo significativo, que tome en cuenta las necesidades de los diferentes contextos rurales, sin descuidar los intereses y expectativas de los jóvenes. Es responsabilidad del sistema educativo brindar una formación de calidad con equidad, pertinencia y relevancia.

\section{REFERENCIAS:}

Achilli, E. (2005). Investigar en Antropología Social. Los desafios de transmitir un oficio, Rosario: Laborde Editor.

Auli, I. (2019). La escuela "no me llama la atención": una reflexión más allá de la escolarización. Ponencia en el XV Congreso Nacional de Investigación Educativa Comie-2019, Acapulco, México.

Ávalos, J. (2007). La vida juvenil en el bachillerato. Una mirada etnográfica. (Tesis de maestría.) DIE-Cinvestav, IPN, México.

Bourdieu, P., y Sayad, A. (2017). El desarraigo. La violencia del capitalismo en una sociedad rural. Argentina: Siglo XXI.

De Ibarrola, M. (2016). Claroscuro en las relaciones entre la escolaridad y el trabajo. Configuraciones y límites. Revista Páginas de Educación, 9(2), 14-48.

Estrada, M. (2007). Del control a la independencia. Género y escolaridad en familias rurales en Guanajuato. En C. Woo y P. Arias (coords.), ¿Campo o ciudad? Nuevos espacios y formas de vida (pp. 45-65). México: Universidad de Guadalajara.

Feito, R. (1990). Nacidos para perder: un análisis sociológico del rechazo y del abandono escolar. España: CIDE. 
Fernández, M. (2011). Del desapego al desenganche y de éste al fracaso escolar. Propuesta educativa, 1(35), 85-94.

Geertz, C. (2001). La interpretación de las culturas. España: Gedisa.

Gessaghi, V., y Sendón, M. A. (2008). Socialización y disposiciones heterogéneas: sus vínculos con la escolarización. Entrevista a Bernard Lahire. Propuesta Educativa, (30), 71-77.

Gimeno, J. (2000). La educación obligatoria: su sentido educativo y social. España: Morata.

Grijalva, O. (2010). Las apariencias como fuente de las identificaciones en la construcción de las identidades y en la conformación de grupos juveniles. (Tesis doctoral.) DIE-Cinvestav, IPN, México.

Glaser, B., y Strauss, A. (1967). The Discovery of grounded theory: strategies for qualitative research. Estados Unidos: Aldine de Gruyter.

Gómez, C., Casares, M., Cifuentes, C., Carmona, A., y Fernández, F. (2001). Identidades de género y feminización del éxito académico. España: Ministerio de Educación, Cultura y Deporte, CIDE.

Guerra, I. (2005). Los jóvenes del siglo XXI, ¿para que trabajan? Los sentidos del trabajo en la vida de jóvenes de sectores urbano-populares de la Ciudad de México. Revista Mexicana de Investigación Educativa, 10(25), 419-449.

Guerra, I., y Guerrero, E. (2004). ¿Qué significado tiene el bachillerato? Una visión desde los jóvenes. México: Universidad Pedagógica Nacional.

Guerrero, E. (2012). Algunos rasgos de la experiencia estudiantil en el bachillerato. En E. Weiss (coord.), Jóvenes y bachillerato (pp. 125-150). México: ANUIES.

Hernández, J. (2008). El trabajo sobre la identidad en estudiantes de bachillerato: reflexividad, voces y marcos morales. México: Universidad Pedagógica Nacional.

INEGI (2010). Censo de población y vivienda 2010. Principales resultados por localidad (ITER). México: Instituto Nacional de Estadística y Geografía.

INEE (2013). Panorama educativo de México 2013. Indicadores del Sistema Educativo Nacional educación básica y media superior. México: INEE.

INEE (2016). Panorama educativo 2016. Indicadores del Sistema Educativo Nacional educación básica y media superior. México: INEE.

Langa, D., y Río, M. (2013). Los estudiantes de clases populares en la universidad frente a la universidad de la crisis: persistencia y nuevas condiciones para la multiplicación de la desigualdad de oportunidades educativas. Tempora, (16), 71-96. 
Miranda, F. (2012). Los jóvenes contra la escuela. Un desafío para pensar las voces y tiempos para América Latina. Revista Latinoamericana de Educación Comparada, 3(3), 71-84.

Muñoz, C. (2006). Determinantes de la empleabilidad de los jóvenes universitarios y alternativas para promoverla. Papeles de Población, 12(49), 75-89.

Pacheco, L. (2016). Para ayudar a mi comunidad. La telesecundaria de la adolescencia indígena. México: Juan Pablos/UAN.

Pérez, L. (2006). Cultura y representaciones de futuro en los estudiantes de la preparatoria EM de Santa Ana Tlacotenco, Milpa Alta, Distrito Federal. (Tesis de maestría.) DIE-Cinvestav, IPN, México.

Reyes, A. (2010). Más allá de los muros. Adolescencias rurales y experiencias estudiantiles en telesecundarias. (Tesis doctoral.) FLACSO, México.

Reyes, A. (2014). Adolescencias rurales, telesecundarias y experiencias estudiantiles. Argumentos, 27(14) 75-93.

Rockwell, E. (2009). La experiencia etnográfica. Historia y cultura en los procesos educativos. Argentina: Paidós.

Saccone, M. (2020). La asistencia a clases de los estudiantes en la educación media superior. Aportes desde una investigación etnográfica en la Ciudad de México. Revista Latinoamericana de Estudios Educativos, $L(2), 55-88$.

Saccone, M., y Weiss, E. (2017). La acreditación de asignaturas en la vida cotidiana escolar. Un estudio etnográfico en el contexto de la obligatoriedad de la educación media superior. Propuesta educativa, (47), 119-128.

Silva, H., y Weiss, E. (2018). Las razones del abandono escolar del bachillerato tecnológico agropecuario. Revista Latinoamericana de Estudios Educativos, XLVIII(1), 73-99.

Tapia, G. (2015). Estudiantes en la Transición Rural-Urbana del Bajío. Los Significados del Bachillerato y del Trabajo. (Tesis doctoral.) DIECinvestav, IPN, México.

Tapia, G. (2019). Mujeres rurales en el bachillerato: la construcción de nuevas identidades aspiracionales. XV Congreso Nacional de Investigación Educativa Comie-2019, Acapulco, México.

Tapia, G., y Weiss, E. (2013). Escuela, trabajo y Familia. Perspectivas de estudiantes de bachillerato en una transición rural-urbana. Revista Mexicana de Investigación Educativa, 18(59), 1165-1188.

Taylor, S. J., y Bogdan, R. (1987). Introducción a los métodos cualitativos de investigación. La búsqueda de significados. España: Paidós. 
Vega, A. (2013). Las conversaciones de los jóvenes en el transporte público. (Tesis de maestría.) DIE-Cinvestav, IPN, México.

Weiss, E. (2006a). Los jóvenes como estudiantes. Revista Mexicana de Investigación Educativa, 11(29), 359-366.

Weiss, E. (2006b). Diagnóstico de las prácticas y procesos curriculares en los bachilleratos integrales comunitarios. México: SEBYN-SEP (Investigación no publicada).

Weiss, E. (Coord.) (2012a). Jóvenes y bachillerato. México: ANUIES.

Weiss, E. (2012b). Los estudiantes como jóvenes. El proceso de subjetivación. Perfiles Educativos, 34(135), 134-148.

Weiss, E. (2017). Hermenéutica y descripción densa versus teoría fundamentada. Revista Mexicana de Investigación Educativa, 22(73), 637-654.

Weiss, E. (2018). Los significados del bachillerato para los jóvenes y la permanencia escolar en México. Sinéctica, (51), 1-19.

Willis, P. (1988). Aprendiendo a trabajar. Cómo los chicos de la clase obrera consiguen trabajos de clase obrera. Espańa: Akal. 